\title{
INFLUENCE OF LOVASTATIN ON PHARMACOKINETICS AND PHARMACODYNAMICS OF GLIPIZIDE IN HEALTHY AND STREPTOZOTOCIN - INDUCED DIABETIC RATS: INVOLVEMENT OF P-GLYCOPROTEIN INHIBITION
}

\author{
SUJATHA SANNEBOINA ${ }^{1,2}$, VANISHREE SAMMETA², RAVINDRA BABU PINGILI ${ }^{2,3}$, KISHORE KUMAR KADIMPATI ${ }^{2,4 *}$ \\ ${ }^{1}$ Department of Pharmaceutics, Narayana Pharmacy College, Chintareddy Palem, Nellore - 524 002, Andhra Pradesh, India. ${ }^{2}$ Department \\ of Pharmacology, Vaagdevi College of Pharmacy, Hanamkonda, Warangal - 506 001, Telangana, India. ${ }^{3}$ Department of Pharmacology, KVSR \\ Siddhartha College of Pharmaceutical Sciences, Vijayawada - 520 010, Andhra Pradesh, India. ${ }^{4}$ Department of Pharmaceutics, Mallareddy \\ College of Pharmacy, Dhullapally, Secunderabad - 500 014, Hyderabad, Telangana, India. Email: drkadimpatikks@gmail.com
}

Received: 07 June 2016, Revised and Accepted: 21 June 2016

\section{ABSTRACT}

This study evaluated the effect of lovastatin (Lov) on the pharmacokinetics and pharmacodynamics of glipizide (Gpz) in healthy and streptozotocininduced diabetic rats. In single dose study (SDS), blood samples were collected on the $1^{\text {st }}$ day, whereas in multiple dose study on the $15^{\text {th }}$ day at 0-12 hrs. Lov significantly altered the pharmacokinetic parameters at the dose of $15 \mathrm{mg} / \mathrm{kg}$ in SDS and multiple dose study. The $\mathrm{C}_{\text {max }}$ of Gpz was increased from 2.97 to 8.38 and 9.87 to $24.58 \mathrm{ng} / \mathrm{mL}$ in healthy and diabetic rats, respectively, in multiple dose study. Rat everted sacs were used to study the transport of Gpz in the presence of Lov and verapamil (P-glycoprotein [P-gp] inhibitor). The transport of Gpz from mucosal to the serosal surface was significantly increased from 4.32 to 5.65 and $6.02 \mu \mathrm{g} / \mathrm{mL}$ in the presence of Lov and verapamil, respectively. The interaction between Lov and Gpz is due to P-gp and CYP2C9 inhibition.

Keywords: Diabetes, Dyslipidemia, Glipizide, Lovastatin, P-glycoprotein.

\section{INTRODUCTION}

Type 2 diabetes mellitus (Type 2 DM) is associated with significant cardiovascular morbidity and mortality. Although low-density lipoprotein cholesterol (LDL-C) levels may be normal in patients with Type 2 DM, insulin resistance drives a number of changes in lipid metabolism and lipoprotein composition that render LDL-C and other lipoproteins more pathogenic than species found in patients without Type 2 DM. Dyslipidemia affects almost $50 \%$ of the patients with Type 2 DM and it is a cardiovascular risk factor [1]. The majority of Indian Type 2 DM patients are dyslipidemic at baseline [2].

The statins are the most effective and best-tolerated agents for treating dyslipidemia. Higher doses of the more potent statins (lovastatin [Lov], atorvastatin, simvastatin, and rosuvastatin) also can reduce triglyceride levels caused by elevated very LDL-C (VLDL) levels. Numerous studies have shown the reduction in cardiovascular morbidity and mortality with statin therapy [3]. Lov is a structural analog of 3-hydroxy-3-methylglutaryl-coenzyme A (HMG CoA) and competitively inhibits HMG-CoA reductase, the rate-limiting enzyme in cholesterol synthesis $[4,5]$. Lov is an inactive lactone prodrug that is hydrolyzed rapidly to the corresponding $\beta$-hydroxy acid metabolite (Lov acid), a potent and competitive inhibitor of HMGCoA reductase $[6,7]$.

Clinically, glipizide (Gpz) is a most widely used next to the metformin in the treatment of Type $2 \mathrm{DM}$, because other sulfonylureas have a higher risk of hypoglycemia especially for glyburide. Gpz is 100 times more potent and is more costly than other sulfonylureas. It is the best buy drug according to Consumer Reports Health Best Buy Drugs [8,9]. The aim of this study was to investigate the influence of Lov on the pharmacokinetics (PK) and pharmacodynamics (PD) of Gpz in healthy and streptozotocin (STZ) induced diabetic rats.

\section{METHODS}

Animals

Male albino rats weighing between 180 and $210 \mathrm{~g}$ were procured from Mahaveer Enterprises, Hyderabad, Andhra Pradesh, India. The animals were housed under standard conditions, maintained on a 12 hrs light/dark cycle. Animals were fasted for $18 \mathrm{hrs}$ before the experiment, and during the experiment, they were withdrawn from food and water. All the experimental protocols were approved by the Institutional Animal Ethical Committee (IAEC). IAEC protocol approval number is 2010/8/1/1-6

\section{Drugs and chemicals}

Lov and Gpz were obtained as a gift samples from Matrix Labs, Hyderabad, Andhra Pradesh, India. Verapamil was obtained from Nicholas Piramal India Ltd., Hyderabad, India. STZ was purchased from Sigma-Aldrich Chemicals Co., St. Louis, MO, USA. Methanol, water (high performance liquid chromatography [HPLC]), potassium dihydrogen orthophosphate, phosphoric acid, diethyl ether, dimethyl sulfoxide purchased from Finar Chemicals Ltd., Ahmedabad, India. All other solvents and chemicals used were of analytical grade.

\section{Methods}

\section{Effect of Lov on the PK of Gpz in healthy rats}

In single dose study (SDS), albino rats were randomly divided into six groups of three animals in each group, i.e., (a) Group I served as control and it received $0.5 \%$ sodium carboxymethylcellulose (SCMC), (b) Group II treated with Lov $(7.5 \mathrm{mg} / \mathrm{kg}$ ), (c) Group III treated Lov (15 mg/kg), (d) Group IV treated with Gpz (5 mg/kg) [10], (e) Group $\mathrm{V}$ treated with Lov $(7.5 \mathrm{mg} / \mathrm{kg})$ followed by Gpz ( $5 \mathrm{mg} / \mathrm{kg}$ ), and (f) Group VI treated with Lov $(15 \mathrm{mg} / \mathrm{kg}$ ) followed by $\mathrm{Gpz}(5 \mathrm{mg} / \mathrm{kg})$.

All the drugs were administered to the respective groups by oral gavages and blood samples were collected from the rats on the $1^{\text {st }}$ day at $0,0.5,1,2,3,4,6,8$ and $12 \mathrm{hrs}$ in SDS. In multi dose study (MDS), same treatment was given for 15 consecutive days and blood samples were collected on the $15^{\text {th }}$ day at same time points like SDS. The plasma was separated by centrifugation (Remi, R - 4C Compact model, Mumbai, India) at $6000 \mathrm{rpm}$ for 6 minutes. The plasma samples were stored at $-20^{\circ} \mathrm{C}$ until analysis. PK parameters were calculated using Kinetica 5.1 software. 
Effect of Lov on the PK of Gpz in STZ - induced diabetic rats Induction of DM

Experimentally diabetes was induced by single intraperitoneal injection of $60 \mathrm{mg} / \mathrm{kg}$ of STZ. STZ was dissolved in freshly prepared cold citrate buffer, $\mathrm{pH} 4.5$ [11]. Control animals received only citrate buffer. After 5 days of STZ injection, animals with fasting blood glucose above $250 \mathrm{mg} / \mathrm{dL}$ were considered as diabetic and included in the study. No adverse effect was observed at the tested concentration throughout the study. The diabetic rats were divided into six groups each consisting of three animals, i.e., (a) Group I served as control and it received 0.5\% SCMC, (b) Group II treated with Lov $(7.5 \mathrm{mg} / \mathrm{kg}$ ) (c) Group III treated Lov (15 mg/kg), (d) Group IV treated with Gpz $(5 \mathrm{mg} / \mathrm{kg}$ ) [10], (e) Group V treated with Lov $(7.5 \mathrm{mg} / \mathrm{kg}$ ) followed by Gpz ( $5 \mathrm{mg} / \mathrm{kg}$ ), and (f) Group VI treated with Lov (15 mg/kg) followed by Gpz $(5 \mathrm{mg} / \mathrm{kg})$.

All the drugs were administered to the respective groups by oral gavages and blood samples were collected from the rats on the $1^{\text {st }}$ day at $0,0.5,1,2,3,4,6,8$ and $12 \mathrm{hrs}$ in SDS. In MDS, same treatment was given for 15 consecutive days and blood samples were collected on the $15^{\text {th }}$ day at same time point like SDS. The plasma was separated by centrifugation (Remi, R-4C Compact model, Mumbai, India) at $6000 \mathrm{rpm}$ for 6 minutes. The plasma samples were stored at $-20^{\circ} \mathrm{C}$ until analysis. PK parameters were calculated using Kinetica 5.1 software.

\section{Extraction of drug from the plasma}

To about $50 \mu \mathrm{L}$ of plasma, $100 \mu \mathrm{L}$ of acetonitrile was added as a protein precipitating agent in eppendorfs tube. The plasma samples were vortexes for 1 minutes and centrifuged at 10,000 rpm for 5 minutes. The supernatant was transferred to another eppendorfs tube and allowed to dryness. The residue was reconstituted with $100 \mu \mathrm{L}$ of the mobile phase and vortex (VWR vortex mixer) for 1 minutes. About $20 \mu \mathrm{L}$ of the reconstituted supernatant was injected onto the reversed phase HPLC (RP-HPLC) system for analysis.

\section{HPLC conditions}

A Shimadzu HPLC system was used to estimate Gpz concentration. It was consisted of a pump (LC-20AT), $\mathrm{C}_{18}$ column (Thermo, $150 \mathrm{~mm} \times 4.6 \mathrm{~mm}, 5 \mu \mathrm{m}$ particle size), and a dual wavelength ultraviolet (UV)-visible detector (SPD-20A). The mobile phase consisted of methanol and phosphate buffer $(70: 30 \mathrm{v} / \mathrm{v})$. The prepared mobile phase was filtered through $0.45 \mu \mathrm{m}$ membrane filter and ultrasonically degassed prior to use. The flow rate was adjusted to $1 \mathrm{~mL} /$ minutes. The effluent was monitored at $230 \mathrm{~nm}$ with a UV detector. The total run of the method was set to be at 6 minutes and the peak area was recorded using LC real time chromatographic data system. The flow rate of $1 \mathrm{~mL} /$ minutes was kept throughout the study. The retention time of Gpz was obtained at 2.90 minutes (Fig. 1).

\section{Intestinal perfusion study}

Preparations of the everted rat gut sacs for intestinal P-glycoprotein (P-gp) activity.

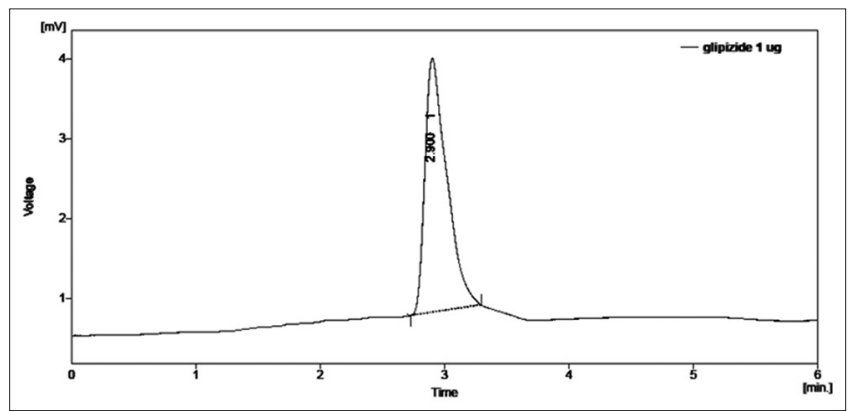

Fig. 1: Chromatogram of glipizide $(1 \mu \mathrm{g})$
P-gp is an energy-dependent transporter protein located in the apical membrane of intestinal mucosal cells. It is believed that it may limit the bioavailability of many orally administered drugs, by transporting them back into the intestinal lumen following their absorption by the enterocytes. The intestinal P-gp activity was demonstrated by Barthe et al. [12] in vitro. We have modified slightly Barthe method; briefly, albino rats (180-220 g) were starved for $24 \mathrm{hrs}$, sacrificed by cervical dislocation and the small intestine removed and washed thoroughly three times with ice cold saline $(0.9 \% \mathrm{NaCl}$ solution). The intestine was immediately placed in oxygenated $\left(\mathrm{O}_{2} / \mathrm{CO}_{2}\right.$, with ratio of $\left.95: 5 \%\right) \mathrm{Krebs}-$ Henseleit solution (KHS) at $37^{\circ} \mathrm{C}$. The composition of KHS is D-glucose, magnesium sulfate, potassium phosphate, potassium chloride, and sodium chloride. The intestine was everted on a glass rod and one end was clamp before filling with KHS. Then, everted sacs were filled with $5 \mathrm{~mL}$ of fresh, oxygenated KHS and sealed with a second clamp. Each sac was placed in individual $50 \mathrm{~mL}$ Erlenmeyer flasks contain $20 \mathrm{~mL}$ of oxygenated KHS and different concentrations of Gpz $(1,10$ and $50 \mu \mathrm{M})$. The sacs were incubated at $37^{\circ} \mathrm{C}$ in an oscillating water bath (15 cycles/minutes) for 30 minutes. After 30 minutes, sacs were removed and washed three times with saline. The sacs were cut open and the serosal fluid (sac contents) was drained into small tubes. The transport of $\mathrm{Gpz}$ from the mucosal to serosal side was determined by RP-HPLC. Other sacs were pre-incubated in $20 \mathrm{~mL}$ of oxygenated KHS containing $100 \mu \mathrm{M}$ of Lov and verapamil in two Erlenmeyer flasks. After 10 minutes, different concentrations of $\operatorname{Gpz}(1,10$, and $50 \mu \mathrm{M})$ were added to the flasks and further incubated at $37^{\circ} \mathrm{C}$ for 30 minutes. Gpz transport across the mucosa was determined by RP-PLC. Each experiment was carried out using the small intestine from one animal.

\section{Determination of blood glucose}

Trinder's glucose oxidase-peroxidase (GOD-POD) method was the most widely used method for estimation of plasma glucose. This method was reported to give satisfactory results with blood samples from normal as well as many pathological conditions of person of all age groups [13]. GOD-POD method reactions as follows:

D-Glucose $+\mathrm{O}_{2}+\mathrm{H}_{2} \mathrm{O} \stackrel{\text { GOD }}{\longrightarrow}$ D- Gluconate $+\mathrm{H}_{2} \mathrm{O}_{2}$

$2 \mathrm{H}_{2} \mathrm{O}_{2}+$ p- hydroxybenzoic acid + 4-aminoantipirine $\stackrel{\text { GOD }}{\longrightarrow}$ Quinoneimine dye $+4 \mathrm{H}_{2} \mathrm{O}$

About $0.1 \mathrm{~mL}$ of blood was added to $2.9 \mathrm{~mL}$ of protein precipitant reagent in a cylindrical centrifuge tube and centrifuged at $2000 \mathrm{rpm}$ for 5 minutes. About $2.5 \mathrm{~mL}$ of clear supernatant fluid was transferred to a test tube labeled as unknown (U). Another two test tubes were also labeled as standard (S) and blank (B). About $2.9 \mathrm{~mL}$ of protein precipitant reagent was placed in $\mathrm{S}$ and $\mathrm{B}$. About $0.1 \mathrm{~mL}$ of water and $0.1 \mathrm{~mL}$ of a standard containing $200 \mathrm{mg}$ of glucose $/ 100 \mathrm{~mL}$ were added to blank (B) and standard (S), respectively. Then, about $0.5 \mathrm{~mL}$ of $0.5 \%$ adrenaline and $0.5 \mathrm{~mL}$ of color reagent were added to test $\mathrm{U}$ and $0.6 \mathrm{~mL}$ quantities of these solutions are added to $S$ and $B$. The tubes were mixed for 40 minutes. The optical densities (OD) of $U$ and $S$ were read at $500 \mathrm{~nm}$ against blank (B) with spectrophotometer (Shimadzu)

Blood glucose $(\mathrm{mg} / 100 \mathrm{~mL})=\frac{\text { OD of Unknown }}{\text { OD of Standard }} \times 100$

Data were expressed as mean \pm standard deviation. The significance was determined by two-way ANOVA followed by post hoc test using GraphPad Prism 5.0 software.

\section{RESULTS}

Effect of Lov on the PK of Gpz in healthy rats

The effect of Lov ( 7.5 and $15 \mathrm{mg} / \mathrm{kg}$ ) on the PK of Gpz was studied in SDS and MDS. In SDS, Lov significantly $(\mathrm{p}<0.001)$ increased the $\mathrm{C}_{\max }$ of Gpz at $4^{\text {th }}$ hrs at the dose of $15 \mathrm{mg} / \mathrm{kg}$, but it has no significant effect at $7.5 \mathrm{mg} / \mathrm{kg}$. The $\mathrm{C}_{\max }$ of $\mathrm{Gpz}$ was increased from $2.97 \pm 0.32$ to $3.07 \pm 0.56$ and $6.38 \pm 0.84 \mathrm{ng} / \mathrm{mL}$ at the dose of 7.5 and $15 \mathrm{mg} / \mathrm{kg}$, respectively. The 
area under the curve (AUC) of Gpz was increased from $20.26 \pm 1.35$ to $35.13 \pm 2.62$ (SDS) and $20.74 \pm 2.35$ to $42.7 \pm 5.38 \mathrm{ng} / \mathrm{mL} / \mathrm{hrs}$ (MDS) in the presence of Lov. Results were shown in Table 1 . Clearance and $V_{d}$ also significantly $(\mathrm{p}>0.05)$ decreased in presence of Lov.

\section{Effect of Lov on the PK of Gpz in STZ-induced diabetic rats}

The effect of Lov studied in the STZ-induced diabetic rats. In diabetic rats, Lov significantly $(\mathrm{p}<0.001)$ increased the $\mathrm{C}_{\mathrm{max}}$ and AUC and decreased clearance and $V_{d}$ of Gpz in SDS and MDS. The results were shown in Table 2

\section{Intestinal perfusion study}

Rat everted sacs were used for intestinal P-gp activity. Everted sacs were incubated at $37^{\circ} \mathrm{C}$ with different concentrations of $\mathrm{Gpz}(1,10$ and $50 \mu \mathrm{M}$ ) in the presence of verapamil (standard P-gp inhibitor) and Lov for 30 minutes. After 30 minutes, Gpz concentration was determined by HPLC in serosal fluid. The transport of Gpz was increased in the presence of verapamil and Lov. Lov significantly $(p<0.001)$ increased the absorption of Gpz like verapamil may be due to P-gp inhibition. The results were depicted in Table 3 .

Effect of Lov on the PD of Gpz in healthy and diabetic rats

The effect of Lov on the PD was studied on the healthy and diabetic rats. Rats were treated with Lov (7.5 and $15 \mathrm{mg} / \mathrm{kg}$ ) followed by Gpz
( $5 \mathrm{mg} / \mathrm{kg}$ ) for 15 days. In SDS, blood glucose levels were estimated on the $1^{\text {st }}$ day and on the $15^{\text {th }}$ day in MDS by GOD-POD method at different time intervals. The maximum glucose reduction was observed at $4^{\text {th }} \mathrm{hrs}$ in both the rats. Results were shown in Tables 4-7. Lov also decreased the blood glucose levels in SDS and MDS, but it is not significant when compared to control. Lov produced synergistic effect on the blood glucose levels with Gpz.

\section{DISCUSSION}

All effective drugs have the potential for producing both benefits and risks associated with desired and undesired effects. The particular response to a drug by a patient is driven in one-way or another by the concentration of that drug and sometimes its metabolites, at the effect sites within the body. Accordingly, it is useful to partition the relationship between drug administration and response into two phases, a pharmacokinetic phase, which relates drug administration to concentrations within the body produced over time and a pharmacodynamic phase, which relates response (desired and undesired) produced to concentration [14].

The cytochrome P450 (CYP450) enzyme family plays a determinant role in the biotransformation of a vast number of structurally diverse drugs. Many drug interactions are a result of the inhibition or induction

Table 1: Pharmacokinetic parameters of Gpz in presence of Lov 7.5 and $15 \mathrm{mg} / \mathrm{kg}$ in healthy rats SDS and MDS

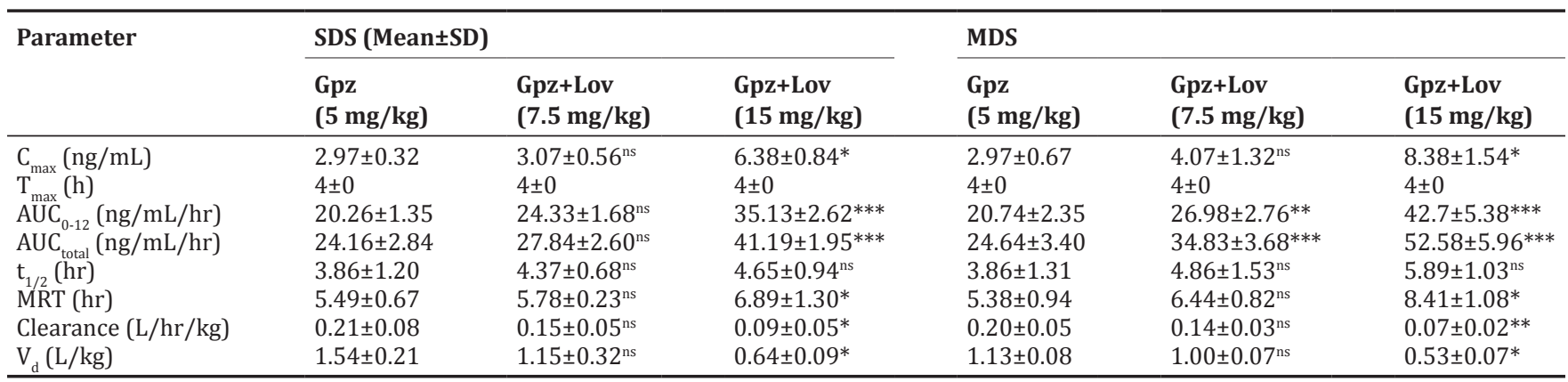

ns: Not significant at $\mathrm{p}>0.05,{ }^{*}$ Significant at $\mathrm{p}<0.05,{ }^{* *}$ Significant at $\mathrm{p}<0.01,{ }^{* * *}$ Significant at $\mathrm{p}<0.001$. SDS: Single dose study, MDS: Multi dose study, Lov: Lovastatin Gpz: Glipizide, AUC: Area under the curve, SD: Standard deviation, MRT: Mean residence time

Table 2: Pharmacokinetic parameters of Gpz in presence of Lov 7.5 and $15 \mathrm{mg} / \mathrm{kg}$ in diabetic rats single dose study and multi dose study

\begin{tabular}{|c|c|c|c|c|c|c|}
\hline \multirow[t]{2}{*}{ Parameter } & \multicolumn{3}{|c|}{ SDS (Mean \pm SD) } & \multicolumn{3}{|c|}{ MDS (Mean \pm SD) } \\
\hline & $\begin{array}{l}\text { Gpz } \\
\text { (5 mg/kg) }\end{array}$ & $\begin{array}{l}\text { Gpz+Lov } \\
\text { (7.5 mg/kg) }\end{array}$ & $\begin{array}{l}\text { Gpz+Lov } \\
\text { (15 mg/kg) }\end{array}$ & $\begin{array}{l}\text { Gpz } \\
\text { (5 mg/kg) }\end{array}$ & $\begin{array}{l}\text { Gpz+Lov } \\
\text { (7.5 mg/kg) }\end{array}$ & $\begin{array}{l}\text { Gpz+Lov } \\
\text { (15 mg/kg) }\end{array}$ \\
\hline $\mathrm{C}_{\max }(\mathrm{ng} / \mathrm{mL})$ & $8.7 \pm 1.58$ & $9.12 \pm 2.13^{\mathrm{ns}}$ & $15.62 \pm 2.64^{*}$ & $9.87 \pm 1.08$ & $12.77 \pm 1.67^{\mathrm{ns}}$ & $24.58 \pm 2.31^{* * *}$ \\
\hline $\mathrm{T}_{\max }^{\max }(\mathrm{hr})$ & $4 \pm 0$ & $4 \pm 0$ & $4 \pm 0$ & $4 \pm 0$ & $4 \pm 0$ & $4 \pm 0$ \\
\hline $\mathrm{AUC}_{0-12}(\mathrm{ng} / \mathrm{mL} / \mathrm{hr})$ & $61.90 \pm 4.51$ & $66.25 \pm 5.62^{\mathrm{ns}}$ & $100.58 \pm 6.70^{* * *}$ & $75.07 \pm 3.57$ & $91.48 \pm 4.62^{* * *}$ & $153.88 \pm 6.85^{* * *}$ \\
\hline $\operatorname{AUC}_{\text {total }}(\mathrm{ng} / \mathrm{mL} / \mathrm{hr})$ & $97.03 \pm 5.97$ & $103.78 \pm 6.71^{\mathrm{ns}}$ & $151.66 \pm 7.15^{* * *}$ & $119.75 \pm 4.25$ & $167.46 \pm 5.60^{* * *}$ & $225.25 \pm 9.63^{* * *}$ \\
\hline $\mathrm{t}_{1 / 2}(\mathrm{hr})$ & $6.49 \pm 0.52$ & $6.58 \pm 0.64^{\mathrm{ns}}$ & $8.40 \pm 0.83^{*}$ & $6.86 \pm 1.21$ & $8.06 \pm 1.39^{\mathrm{ns}}$ & $9.60 \pm 0.95^{*}$ \\
\hline MRT (hr) & $6.17 \pm 0.54$ & $7.14 \pm 0.31^{\mathrm{ns}}$ & $8.51 \pm 0.36^{*}$ & $6.23 \pm 0.98$ & $7.14 \pm 0.75^{\mathrm{ns}}$ & $9.85 \pm 0.86^{*}$ \\
\hline Clearance (L/hr/kg) & $0.05 \pm 0.001$ & $0.048 \pm 0.00^{\mathrm{ns}}$ & $0.03 \pm 0.001^{*}$ & $0.04 \pm 0.001$ & $0.03 \pm 0.001^{\mathrm{ns}}$ & $0.02 \pm 0.001 *$ \\
\hline $\mathrm{V}_{d}(\mathrm{~L} / \mathrm{kg})$ & $0.48 \pm 0.02$ & $0.45 \pm 0.02^{\mathrm{ns}}$ & $0.30 \pm 0.02 *$ & $0.41 \pm 0.02$ & $0.39 \pm 0.01^{\mathrm{ns}}$ & $0.12 \pm 0.02 *$ \\
\hline
\end{tabular}

ns: Not significant at $\mathrm{p}>0.05,{ }^{*}$ Significant at $\mathrm{p}<0.05,{ }^{* * *}$ Significant at $\mathrm{p}<0.001$. SDS: Single dose study, MDS: Multi dose study, Lov: Lovastatin, Gpz: Glipizide, AUC: Area under the curve, SD: Standard deviation, MRT: Mean residence time

Table 3: Transport of glipizide $(\mu \mathrm{g} / \mathrm{mL})$ from mucosal to serosal side in the presence and absence of verapamil and lovastatin ( $\mathrm{n}=3 \mathrm{sacs})$

\begin{tabular}{llll}
\hline $\begin{array}{l}\text { Glipizide concentration } \\
\text { in mucosal side }(\boldsymbol{\mu M})\end{array}$ & $\begin{array}{l}\text { Glipizide concentration } \\
\text { in serosal side }(\boldsymbol{\mu g})\end{array}$ & $\begin{array}{l}\text { Glipizide concentration in } \\
\text { serosal side (ng) in presence } \\
\text { of lovastatin }(\mathbf{1 0 0} \boldsymbol{\mu M})\end{array}$ \\
\hline 1 & $1.35 \pm 0.02$ & $3.39 \pm 0.02^{* * *}$ & $\begin{array}{l}\text { Glipizide concentration in } \\
\text { serosal side }(\mathbf{n g}) \text { in presence } \\
\text { of verapamil }(\mathbf{1 0 0} \boldsymbol{\mu M})\end{array}$ \\
10 & $2.75 \pm 0.16$ & $4.11 \pm 0.26^{* * *}$ & $4.45 \pm 0.26^{* * *}$ \\
50 & $4.32 \pm 0.34$ & $5.65 \pm 0.38^{* * *}$ & $6.02 \pm 0.30^{* * *}$ \\
\hline
\end{tabular}

***Significant at $\mathrm{p}<0.001$ compared to glipizide control 
Table 4: \% reduction of blood glucose after oral administration of Gpz, Lov and their combinations in healthy rats (SDS)

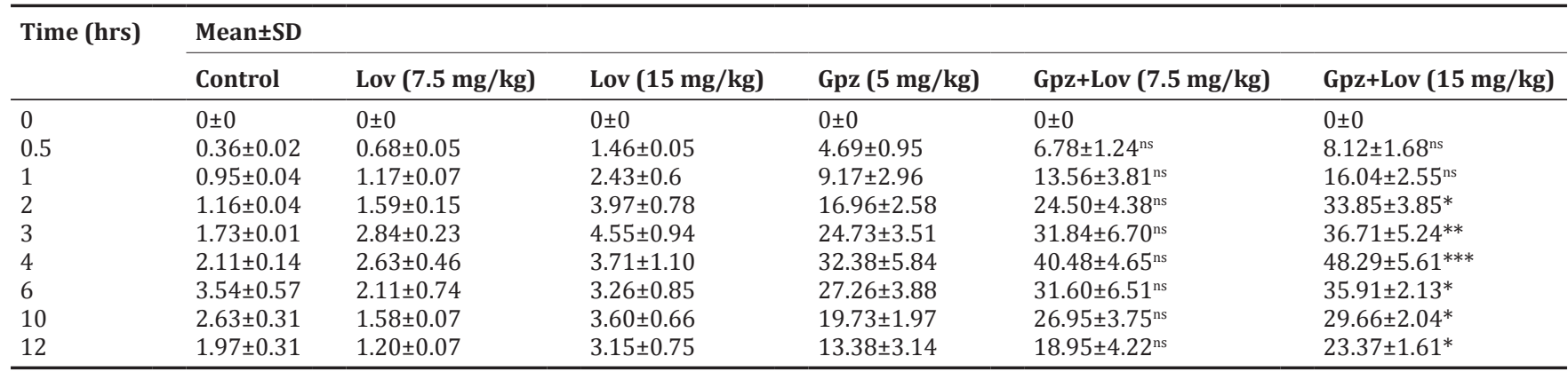

ns: Not significant at $\mathrm{p}>0.05$, *Significant at $\mathrm{p}<0.05,{ }^{* *}$ Significant at $\mathrm{p}<0.01,{ }^{* * *}$ Significant at $\mathrm{p}<0.001$. SDS: Single dose study, Lov: Lovastatin, Gpz: Glipizide, SD: Standard deviation

Table 5: \% reduction of blood glucose after oral administration of Gpz, Lov and their combinations in healthy rats (MDS)

\begin{tabular}{|c|c|c|c|c|c|c|}
\hline \multirow[t]{2}{*}{ Time (hrs) } & \multicolumn{6}{|l|}{ Mean \pm SD } \\
\hline & Control & Lov (7.5 mg/kg) & Lov (15 mg/kg) & Gpz (5 mg/kg) & Gpz+Lov $(7.5 \mathrm{mg} / \mathrm{kg})$ & Gpz+Lov $(15 \mathrm{mg} / \mathrm{kg})$ \\
\hline 0 & $0 \pm 0$ & $0 \pm 0$ & $0 \pm 0$ & $0 \pm 0$ & $0 \pm 0$ & $0 \pm 0$ \\
\hline 0.5 & $0.85 \pm 0.05$ & $1.32 \pm 0.07$ & $1.80 \pm 0.31$ & $6.24 \pm 0.12$ & $7.75 \pm 1.55^{\mathrm{ns}}$ & $10.37 \pm 1.89^{\text {ns }}$ \\
\hline 1 & $1.48 \pm 0.05$ & $1.83 \pm 0.23$ & $2.75 \pm 0.65$ & $12.71 \pm 1.36$ & $18.26 \pm 4.80^{\text {ns }}$ & $25.19 \pm 3.25^{* *}$ \\
\hline 2 & $1.93 \pm 0.25$ & $2.70 \pm 0.52$ & $4.63 \pm 1.04$ & $20.60 \pm 2.58$ & $27.65 \pm 4.58^{\text {ns }}$ & $38.47 \pm 1.65^{* * *}$ \\
\hline 3 & $2.66 \pm 0.43$ & $3.78 \pm 0.64$ & $6.51 \pm 1.67$ & $28.97 \pm 3.62$ & $38.55 \pm 6.75^{*}$ & $47.67 \pm 5.34^{* * *}$ \\
\hline 4 & $3.07 \pm 0.75$ & $2.53 \pm 0.85$ & $5.33 \pm 1.22$ & $39.86 \pm 5.37$ & $50.16 \pm 7.63^{*}$ & $61.49 \pm 6.81^{* * *}$ \\
\hline 6 & $6.42 \pm 1.94$ & $5.72 \pm 1.04$ & $4.61 \pm 1.24$ & $31.28 \pm 2.18$ & $37.94 \pm 6.32^{\text {ns }}$ & $43.25 \pm 4.83^{* *}$ \\
\hline 10 & $4.13 \pm 1.05$ & $4.65 \pm 1.1$ & $5.57 \pm 1.60$ & $22.75 \pm 2.26$ & $26.63 \pm 4.71^{\text {ns }}$ & $35.94 \pm 4.34^{* *}$ \\
\hline 12 & $3.51 \pm 1.10$ & $4.44 \pm 0.84$ & $3.53 \pm 0.55$ & $15.11 \pm 2.5$ & $21.54 \pm 3.02^{\mathrm{ns}}$ & $26.13 \pm 2.61^{*}$ \\
\hline
\end{tabular}

Ns: Not significant at $\mathrm{p}>0.05,{ }^{*}$ Significant at $\mathrm{p}<0.05,{ }^{*}$ Significant at $\mathrm{p}<0.01,{ }^{* * *}$ Significant at $\mathrm{p}<0.001$. MDS: Multi dose study, Lov: Lovastatin, Gpz: Glipizide, SD: Standard deviation

Table 6: \% reduction of blood glucose after oral administration of Gpz, Lov and their combinations in diabetic rats (SDS)

\begin{tabular}{|c|c|c|c|c|c|c|}
\hline \multirow[t]{2}{*}{ Time (hrs) } & \multicolumn{6}{|l|}{ Mean \pm SD } \\
\hline & Control & Lov (7.5 mg/kg) & Lov (15 mg/kg) & Gpz (5 mg/kg) & Gpz+Lov (7.5 mg/kg) & Gpz+Lov (15 mg/kg) \\
\hline 0 & $0 \pm 0$ & $0 \pm 0$ & $0 \pm 0$ & $0 \pm 0$ & $0 \pm 0$ & $0 \pm 0$ \\
\hline 1 & $2.42 \pm 0.42$ & $2.68 \pm 0.61$ & $3.32 \pm 0.87$ & $11.38 \pm 2.63$ & $20.20 \pm 3.58$ & $28.77 \pm 3.22^{\mathrm{ns}}$ \\
\hline 2 & $2.87 \pm 0.48$ & $3.08 \pm 0.55$ & $5.74 \pm 1.07$ & $21.33 \pm 3.76$ & $34.14 \pm 4.72$ & $43.30 \pm 4.63^{* * *}$ \\
\hline 3 & $3.50 \pm 0.63$ & $4.76 \pm 1.06$ & $7.55 \pm 1.64$ & $29.20 \pm 6.45$ & $43.61 \pm 5.62^{* *}$ & $52.45 \pm 6.40^{* * *}$ \\
\hline 4 & $3.85 \pm 0.87$ & $4.22 \pm 0.74$ & $6.10 \pm 1.53$ & $38.87 \pm 8.25$ & $53.50 \pm 6.33^{* *}$ & $76.17 \pm 5.63^{* * *}$ \\
\hline 6 & $4.06 \pm 1.30$ & $3.59 \pm 1.10$ & $5.33 \pm 0.95$ & $27.57 \pm 3.33$ & $36.43 \pm 6.21^{* *}$ & $61.44 \pm 6.75^{* * *}$ \\
\hline 10 & $4.15 \pm 1.02$ & $3.40 \pm 0.81$ & $4.26 \pm 0.55$ & $21.70 \pm 3.64$ & $30.13 \pm 3.20$ & $45.36 \pm 3.41^{* * *}$ \\
\hline 12 & $3.54 \pm 0.95$ & $3.13 \pm 0.81$ & $4.00 \pm 0.73$ & $16.14 \pm 3.12$ & $21.32 \pm 2.35$ & $32.55 \pm 3.50^{* * *}$ \\
\hline
\end{tabular}

ns: Not significant at $\mathrm{p}>0.05,{ }^{* *}$ Significant at $\mathrm{p}<0.01,{ }^{* * *}$ Significant at $\mathrm{p}<0.001$. SDS: Single dose study, Lov: Lovastatin, Gpz: Glipizide, SD: Standard deviation

of CYP enzymes. P-gp, the most extensively studied ATP-binding cassette transporter, functions as a biological barrier by extruding toxic substances and xenobiotics out of cells. In vitro and in vivo studies have demonstrated that P-gp plays a significant role in drug absorption and disposition. Like CYP450 enzymes, inhibition and induction of P-gp have been reported as the causes of drug-drug interactions. Because many prototypic inhibitors and inducers affect both CYP3A4 and P-gp, many drug interactions caused by these inhibitors and inducers involve these two systems. Clinically, it is very difficult to quantitatively differentiate P-gp mediated drug interactions versus CYP3A4 mediated drug interactions $[15,16]$.

Lov is incompletely absorbed from the gastrointestinal (GI) tract and undergoes extensive first-pass extraction in the liver [17]. Lov is a substrate of CYP3A4 hepatic metabolism but does not affect the metabolism of CYP3A4 substrates. Both Lov and its beta-hydroxy acid metabolite are highly bound (>95\%) to plasma proteins [18]. Gpz is rapidly and completely absorbed from the GI tract. Gpz is distributed within the extracellular fluid and is highly protein-bound (99\%). Gpz is extensively metabolized in the liver (approximately 90\%) to inactive metabolites and both unchanged drug and metabolites are excreted in the urine. Gpz is a CYP2C9 substrate, and theoretically CYP2C9 inhibitors or inducers may affect its metabolism [19]. Bosentan may induce CYP2C9 metabolism of Gpz based on theoretical cytochrome P-450 interactions and it decreases the plasma concentration of Gpz. Sulfinpyrazone is an inhibitor of CYP2C9 and serum glucose concentrations monitoring is required if $\mathrm{Gpz}$ is co administered with sulfinpyrazone [20]. In this study, the plasma concentration and AUC of Gpz were significantly ( $\mathrm{p}<0.001)$ increased with Lov $(15 \mathrm{mg} / \mathrm{kg})$ in both healthy and diabetic rats in SDS and MDS at $4^{\text {th }}$ hrs. The results were shown in Figs. 2 and 3.

Fluvastatin selectively inhibits a major drug metabolizing enzyme (CYP2C9). Lov and simvastatin also inhibits the liver oxidation of CYP2C9 substrates [21]. The results suggested that Lov is a weak inhibitor of CYP2C9; thereby it increased the $\mathrm{C}_{\max }$ and AUC of Gpz (CYP2C9 substrate). In this study, rat everted intestinal sacs were also used to study the effect of Lov on the intestinal transport of $\mathrm{Gpz}$ and P-gp activity. The transport of Gpz from mucosal to serosal side was increased in the presence of Lov and verapamil (standard P-gp 
Table 7: \% reduction of blood glucose after oral administration of $\mathrm{Gpz}$, Lov and their combinations in diabetic rats (MDS)

\begin{tabular}{|c|c|c|c|c|c|c|}
\hline \multirow[t]{2}{*}{ Time (hrs) } & \multicolumn{6}{|l|}{ Mean \pm SD } \\
\hline & Control & Lov (7.5 mg/kg) & Lov (15 mg/kg) & Gpz (5 mg/kg) & Gpz+Lov (7.5 mg/kg) & Gpz+Lov (15 mg/kg) \\
\hline 0 & $0 \pm 0$ & $0 \pm 0$ & $0 \pm 0$ & $0 \pm 0$ & $0 \pm 0$ & $0 \pm 0$ \\
\hline 1 & $2.75 \pm 0.77$ & $3.13 \pm 0.49$ & $3.57 \pm 0.61$ & $17.72 \pm 2.15$ & $32.13 \pm 2.50^{* *}$ & $40.90 \pm 3.44^{* * *}$ \\
\hline 2 & $3.58 \pm 0.56$ & $4.34 \pm 0.87$ & $5.89 \pm 1.09$ & $28.08 \pm 2.46$ & $41.66 \pm 4.38^{* *}$ & $53.63 \pm 4.65^{* * *}$ \\
\hline 3 & $5.20 \pm 1.30$ & $5.85 \pm 1.32$ & $7.04 \pm 1.51$ & $41.70 \pm 6.75$ & $57.18 \pm 6.92^{* *}$ & $75.84 \pm 6.86^{* * *}$ \\
\hline 4 & $5.31 \pm 1.22$ & $6.83 \pm 1.57$ & $8.32 \pm 1.69$ & $53.66 \pm 7.10$ & $72.39 \pm 8.13^{* * *}$ & $85.51 \pm 7.83^{* * *}$ \\
\hline 6 & $5.88 \pm 1.52$ & $6.43 \pm 1.95$ & $7.55 \pm 1.22$ & $36.29 \pm 5.91$ & $53.64 \pm 7.50^{* * *}$ & $69.25 \pm 6.55^{* * *}$ \\
\hline 10 & $4.53 \pm 0.75$ & $5.31 \pm 0.66$ & $5.90 \pm 0.87$ & $25.84 \pm 4.0$ & $38.95 \pm 4.15^{*}$ & $51.54 \pm 4.97^{* * *}$ \\
\hline 12 & $4.21 \pm 0.79$ & $4.66 \pm 0.93$ & $4.31 \pm 0.56$ & $18.38 \pm 2.43$ & $26.34 \pm 3.53^{\mathrm{ns}}$ & $35.44 \pm 3.11^{* * *}$ \\
\hline
\end{tabular}

ns: Not significant at $p>0.05$, *Significant at $p<0.05,{ }^{* *}$ Significant at $p<0.01,{ }^{* * *}$ Significant at $p<0.001$. MDS: Multi dose study, Lov: Lovastatin, Gpz: Glipizide, SD: Standard deviation

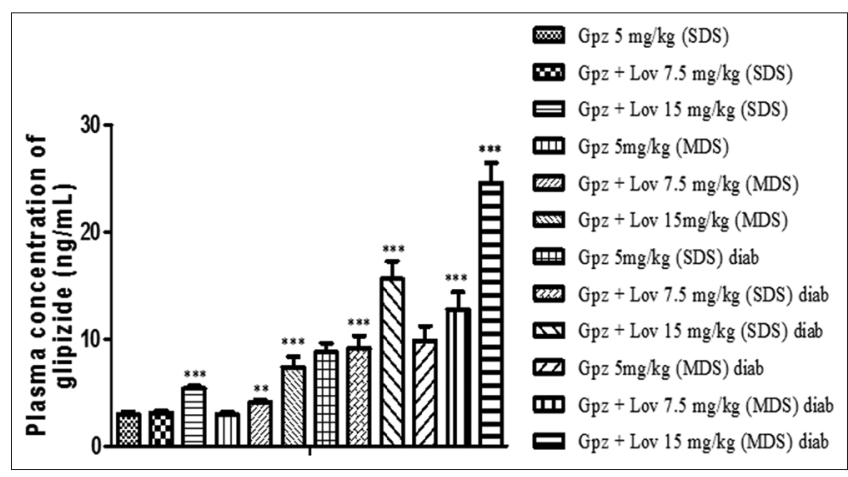

Fig. 2: The plasma concentration of glipizide in presence of lovastatin in healthy and diabetic rats in single dose study and multi dose study

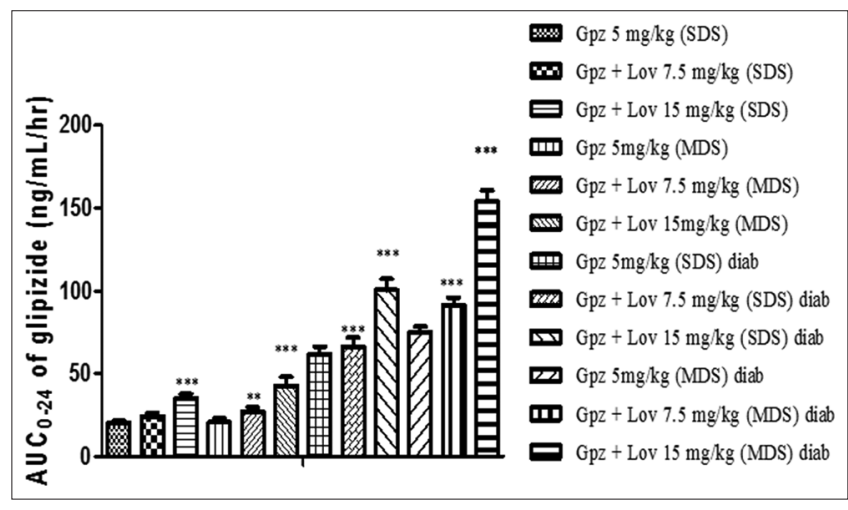

Fig. 3: The area under the curve of glipizide in presence of lovastatin in healthy and diabetic rats in single dose study and multi dose study

inhibitor). Lov is a P-gp substrate [22] and it also a potent inhibitor of P-gp [23-25]. Results were shown in Fig. 4.

In another study, Lov increased the AUC and $\mathrm{C}_{\max }$ of repaglinide in rats and rabbits due to P-gp and CYP3A4 inhibition [26]. Gpz is a P-gp substrate [10]; thereby Lov increased the plasma concentration of Gpz. In PD study, Lov reduced the maximum glucose levels at $4^{\text {th }}$ hrs in healthy and diabetic rats in SDS and MDS. Lov also has some hypoglycemic activity but it is not significant when compared with control. Synergistic effect was observed on the blood glucose levels with this combination (Tables 4-7).

\section{CONCLUSIONS}

Statins are the first choice drugs for the treatment of elevated LDL-C in Type 2 DM. Gpz is the second generation sulfonylurea. It is 100 times

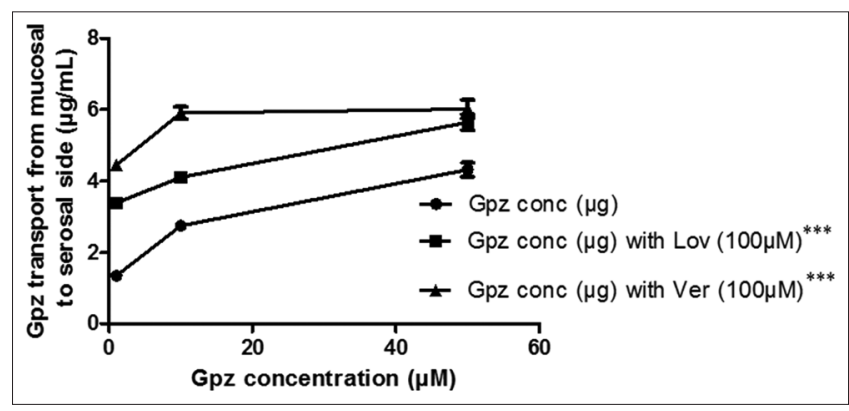

Fig. 4: Transport of glipizide from mucosal to serosal side in presence of lovastatin and verapamil

more potent and is more costly than other sulfonylureas. The maximum dose of Gpz is $40 \mathrm{mg} /$ day. The combinations of these drugs are used to treat hypercholesterolemia in patients with Type $2 \mathrm{DM}$. Lov is a potent P-gp inhibitor. It also has weak inhibitory effect on CYP2C9; thereby, it increases the bioavailability of Gpz. Dosage adjustment of Gpz is needed. Continuous monitoring of glucose concentration is required with this combination due to the risk of hypoglycemia.

\section{ACKNOWLEDGMENTS}

Authors are very grateful to Matrix Lab, Hyderabad for providing lovastatin and Glipizide as gift samples.

\section{REFERENCES}

1. Vijayaraghavan K. Treatment of dyslipidemia in patients with Type 2 diabetes. Lipids Health Dis 2010;9:144.

2. Parikh RM, Joshi SR, Menon PS, Shah NS. Prevalence and pattern of diabetic dyslipidemia in Indian Type 2 diabetic patients. Diabetes Metab Syndr Clin Res Rev 2010;4(1):10-2.

3. Blumenthal D, Buxton I, Parker K. Goodman \& Gilman's. Manual of Pharmacology and Therapeutics. New York: McGraw- Hill; 2007. p. 611 .

4. Illingworth DR, Tobert JA. A review of clinical trials comparing HMG-CoA reductase inhibitors. Clin Ther 1994;16(3):366-85.

5. Vaughan CJ, Murphy MB, Buckley BM. Statins do more than just lower cholesterol. Lancet 1996;348(9034):1079-82.

6. Tobert JA. New developments in lipid-lowering therapy: The role of inhibitors of hydroxymethylglutaryl-coenzyme A reductase. Circulation 1987;76(3):534-8

7. Alberts AW. Discovery, biochemistry and biology of lovastatin. Am J Cardiol 1988;62(15):10J-15

8. Bennett WL, Maruthur NM, Singh S, Segal JB, Wilson LM, Chatterjee R, et al. Comparative effectiveness and safety of medications for Type 2 diabetes: An update including new drugs and 2-drug combinations. Ann Intern Med 2011;154(9):602-13.

9. Bennett WL. Oral diabetes medications for adults with Type 2 diabetes: An update. Comparative Effectiveness Review No.27; 2011.

10. Ravindra BP, Vanishree S, Sujatha S, Kishore KK. Effect of carvedilol on the pharmacokinetics and pharmacodynamics of glipizide. Int J Pharm Pharm Sci 2012;4(3):133-8. 
11. Pandit R, Phadke A, Jagtap A. Antidiabetic effect of Ficus religiosa extract in streptozotocin-induced diabetic rats. J Ethnopharmacol 2010;128(2):462-6.

12. Barthe L, Bessouet M, Woodley JF, Houin G. The improved everted gut sac: A simple method to study intestinal P-glycoprotein. Int J Pharm 1998;173(1):255-8.

13. Trinder P. Determination of blood glucose using an oxidaseperoxidase system with a non-carcinogenic chromogen. J Clin Pathol 1969;22(2):158-61

14. David Rodrigues A. Drug-Drug Interactions. $2^{\text {nd }}$ ed. USA: Informa Healthcare; 2008

15. Wadhwa NK, Schroeder TJ, Pesce AJ, Myre SA, Clardy CW, First MR. Cyclosporine drug interactions: A review. Ther Drug Monit 1987;9(4):399-406.

16. Whitlock JP, Denison MS. Induction of cytochrome P450 enzymes that metabolize xenobiotics. $2^{\text {nd }}$ ed. New York: Plenum Press; 1995. p. 367-90.

17. Davidson MH, Lukacsko P, Sun JX, Phillips G, Walters E, Sterman A, et al. A multiple-dose pharmacodynamic, safety, and pharmacokinetic comparison of extended- and immediate-release formulations of lovastatin. Clin Ther 2002;24(1):112-25.

18. Williams D, Feely J. Pharmacokinetic-pharmacodynamic drug interactions with $\mathrm{HMG}-\mathrm{Co} A$ reductase inhibitors. Clin Pharmacokinet 2002;41(5):343-70

19. Kirchheiner J, Roots I, Goldammer M, Rosenkranz B, Brockmöller J Effect of genetic polymorphisms in cytochrome p450 (CYP) 2C9 and CYP2C8 on the pharmacokinetics of oral antidiabetic drugs: Clinical relevance. Clin Pharmacokinet 2005;44(12):1209-25.

20. Hansten PD, Horn JR. Cytochrome P450 enzymes and drug interactions, table of cytochrome $\mathrm{P} 450$ substrates, inhibitors, inducers and P-glycoprotein, with footnotes. In: The Top 100 Drug Interactions - A guide to Patient Management. Freeland, WA: H\&H Publications; 2008. p. $142-57$.

21. Transon C, Leemann T, Dayer P. In vitro comparative inhibition profiles of major human drug metabolising cytochrome $\mathrm{P} 450$ isozymes (CYP2C9, CYP2D6 and CYP3A4) by HMG-CoA reductase inhibitors. Eur J Clin Pharmacol 1996;50(3):209-15.

22. Sakaeda T, Takara K, Kakumoto M, Ohmoto N, Nakamura T, Iwaki K, et al. Simvastatin and lovastatin, but not pravastatin, interact with MDR1. J Pharm Pharmacol 2002;54(3):419-23.

23. Bogman K, Peyer AK, Török M, Küsters E, Drewe J. HMG-CoA reductase inhibitors and P-glycoprotein modulation. Br J Pharmacol 2001;132(6):1183-92

24. Choi DH, Chung JH, Choi JS. Pharmacokinetic interaction between oral lovastatin and verapamil in healthy subjects: Role of P-glycoprotein inhibition by lovastatin. Eur J Clin Pharmacol 2010;66(3):285-90.

25. Hong SP, Yang JS, Han JY, Ha SI, Chung JW, Koh YY, et al. Effects of lovastatin on the pharmacokinetics of diltiazem and its main metabolite, desacetyldiltiazem, in rats: Possible role of cytochrome P450 3A4 and P-glycoprotein inhibition by lovastatin. J Pharm Pharmacol 2011;63(1):129-35

26. Makula CS, Reddy PJ. Influence of lovastatin on the pharmacodynamic and pharmacokinetic activity of repaglinide in rats and rabbits. J Pharm Res 2012;5(4):2018-21 